\title{
Quantum Anti-de Sitter Space
}

\author{
Zhe Chang \\ Max-Planck-Institut für Physik \\ Werner-Heisenberg-Institut \\ Föhringer Ring 6, D-80805 München, Germany \\ and \\ Institute of High Energy Physics, Academia Sinica \\ P.O.Box 918(4), 100039 Beijing, China
}

\begin{abstract}
The quantum Anti-de Sitter (AdS) group and quantum AdS space is discussed. Ways of getting the quantum AdS group from real forms of quantum orthogonal group are presented. Differential calculus on the quantum AdS space are also introduced. In particular, reality of differential calculus are given. We set up explicit relationships between quantum group and quantum algebra, which can be refereed as the quantum counterpart of the classical exponential. By this way, quantum AdS algebra is deduced from conjugation on the quantum AdS group.
\end{abstract}




\section{Introduction}

The classical Anti-de Sitter (AdS) space can be defined as the four-dimensional hyperboloid

$$
\eta_{a b} x^{a} x^{b}=-\frac{1}{a^{2}}
$$

in $R^{5}$ with Cartesian coordinates $x^{a}$, where $\eta_{a b}=\operatorname{diag}(-1,1,1,1,-1)$. In polar coordinates $y^{\mu}=(t, r, \theta, \phi)$ the line element may be written

$$
g_{\mu \nu} d y^{\mu} d y^{\nu}=-\left(1+a^{2} r^{2}\right) d t^{2}+\left(1+a^{2} r^{2}\right)^{-1} d r^{2}+r^{2}\left(d \theta^{2}+\sin ^{2} \theta d \phi^{2}\right) .
$$

The symmetry group (isometry group) of the classical AdS space is $S O(3,2)$, which plays the role of the Poincaré group on Minkowski space. In fact, the Poincaré group can be obtained from $S O(3,2)$ by a contraction. The AdS space has rather peculiar features. Its time-like geodesics are finite and closed. The time translations are a $U(1)$ subgroup of $S O(3,2)$. The space-like geodesics are unbounded. Furthermore the casual structure is somewhat complicated.

The AdS group, AdS space and quantum field theory based on it has been an interested topic for a long time[1]. In the early 80's, there was great interest in fourdimensional $N$-extended supergravities for which the global $S O(N)$ is promoted to a gauge symmetry[2]. In these theories the underlying supersymmetry algebra is no longer Poincaré but rather AdS. An important ingredient in these developments was that the $\mathrm{AdS} \times S^{7}$ geometry was not fed in by hand but resulted from a spontaneous compactification, i.e., the vacuum state was obtained by finding a stable solution of the higher-dimensional field equations. There has recently been a revival of interest in AdS space brought about by the conjectured duality between physics in the bulk of AdS and a conformal field theory on the boundary[3]. This is one of the most important progresses in the so-called non-perturbative superstring theory. Many new results have been obtained by making use this conjecture田[5]. Here the AdS geometry plays a central role. It might seem very strange that quantum theories in different space-time dimensions could be equivalent. This possibility is related to the fact that the theory in the large dimension is (among other things) a quantum theory of gravity. For such 
theories the concept of holography has been introduced as a generic property. The Maldacena conjecture is an example of realization of the holography.

According to general relativity, gravity is nothing but space-time geometry. The poor understanding of physics at very short distances indicates that the small scale structure of space-time might not be adequately described by classical continuum geometry. It is long been suspected that the noncommutivity of space-time might be a realistic picture of how space-time behaves near the Planck scale where strong quantum fluctuations of gravity may make points in space fuzzy. As it is well known, superstring theory is now the only possible candidate for quantum theory of gravity [6]. The recent efforts to unify by non-perturbative dualities all the known five perturbative superstring theory is referred as M theory [7]. Many physicists believe that the M theory is a fundamental quantum theory in eleven dimensional space-time. The BFSS matrix model $[8]$ is proposed for the microscopic description of $\mathrm{M}$ theory in discrete light-cone quantization. The basic block in the matrix model is a set of $N$ partons, called $D_{0^{-}}$ branes, on which strings can end. A novel feature of the M(atrix) theory is that the nine transverse coordinates of the $D_{0}$-branes are promoted into $N \times N$ Hermitian matrices. Then the noncommutative geometry becomes a natural way to deal with spaces whose coordinates are noncommutative.

Because the noncommutative geometry description is a strong candidate of realistic pictures of space-time behaviors at Planck scale and the Madacena conjecture a concrete realization of holography -the generic feature of quantum theory of gravity, it is nature to assume that the proper geometry for the Madacena conjecture be the noncommutative AdS space.

In this paper, we discuss the quantum AdS space and quantum AdS group by investigating real forms of quantum orthogonal group and quantum orthogonal space. Differential calculus on the quantum AdS space are presented. In particular, we set up relationships between quantum group and quantum algebra, which may be referred as the quantum counterpart of the classical exponential. It is the relationship which deduces the quantum AdS algebra naturally from the conjugation operation on the 
quantum AdS group. The presentations of the quantum AdS algebra are foundations for constructing quantum field theory on the quantum AdS space.

This paper is organized as follows. In Section 2, we present some preliminary knowledge about quantum group theory and differential calculus on quantum orthogonal space. A realization of the quantum algebra $U_{q}(s o(5))$ in quantum orthogonal space is shown in Section 3. In Section 4, kinds of conjugations on quantum orthogonal group and quantum orthogonal space are constructed to get quantum AdS group and quantum AdS space both for $|q|=1$ and $q$ being real cases. We state the differential calculus on quantum AdS space in Section 5. Reality of differential calculus is discussed. To deduce quantum AdS algebra from conjugation on quantum orthogonal space and quantum orthogonal group, we set up explicit relationships between quantum group $S O_{q}(5)$ and quantum algebra $U_{q}(s o(5))$ in Section 6. Section 7 is devoted to study of quantum AdS algebra.

\section{Differential calculus on quantum space}

The quantum $\mathcal{R}$ matrix 9] for the quantum group $S O_{q}(5)$ is of the form

$$
\begin{aligned}
\mathcal{R}= & q \sum_{\substack{i=-2 \\
i \neq 0}}^{2} e_{i}^{i} \otimes e_{i}^{i}+\sum_{\substack{i, j=-2 \\
i \neq j, j j^{\prime} \\
\text { or } i=j=0}}^{2} e_{i}^{i} \otimes e_{j}^{j}+q^{-1} \sum_{\substack{i=-2 \\
i \neq 0}}^{2} e_{i^{\prime}}^{i^{\prime}} \otimes e_{i}^{i} \\
& +\lambda\left[\sum_{\substack{i, j=-2 \\
i>j}}^{2} e_{j}^{i} \otimes e_{i}^{j}-\sum_{\substack{i, j=-2 \\
i>j}}^{2} q^{\rho_{i}-\rho_{j}} e_{j}^{i} \otimes e_{j^{\prime}}^{i^{\prime}}\right]
\end{aligned}
$$

where $\lambda=q-q^{-1}, i^{\prime}=-i$ and $\rho_{i}=\left(\frac{3}{2}, \frac{1}{2}, 0,-\frac{1}{2},-\frac{3}{2}\right)$.

By making use of the $\mathcal{R}$ matrix, the quantum group $S O_{q}(5)$ with entries

$$
T=\left(\begin{array}{ccccc}
t_{11} & t_{12} & t_{13} & t_{14} & t_{15} \\
t_{21} & t_{22} & t_{23} & t_{24} & t_{25} \\
t_{31} & t_{32} & t_{33} & t_{34} & t_{35} \\
t_{41} & t_{42} & t_{43} & t_{44} & t_{45} \\
t_{51} & t_{52} & t_{53} & t_{54} & t_{55}
\end{array}\right)
$$

can be written into the standard form 


$$
\mathcal{R} T_{1} T_{2}=T_{2} T_{1} \mathcal{R},
$$

where $T_{1} \equiv T \otimes 1$ and $T_{2} \equiv 1 \otimes T$.

Of course, the $T$ matrix also should satisfy the orthogonal relations

$$
T^{t} C T=C, \quad T C T^{t}=C,
$$

where $C_{i j}$ is the metric on the quantum orthogonal space,

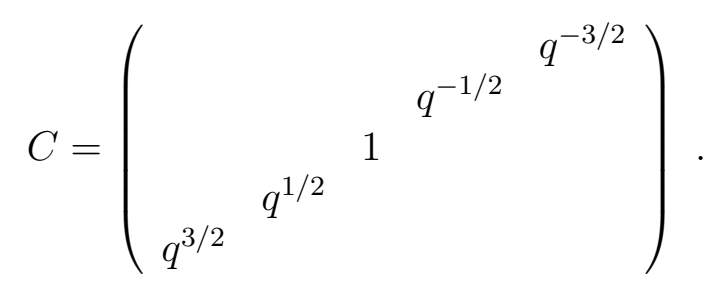

The Hopf algebra structure of the quantum orthogonal group is

$$
\begin{aligned}
& \Delta(T)=T \dot{\otimes} T, \\
& \epsilon(T)=I \\
& S(T)=C T^{t} C^{-1}=\left(\begin{array}{ccccc}
t_{55} & q^{-1} t_{45} & q^{-3 / 2} t_{35} & q^{-2} t_{25} & q^{-3} t_{15} \\
q t_{54} & t_{44} & q^{-1 / 2} t_{34} & q^{-1} t_{24} & q^{-2} t_{14} \\
q^{3 / 2} t_{53} & q^{1 / 2} t_{43} & t_{33} & q^{-1 / 2} t_{23} & q^{-3 / 2} t_{13} \\
q^{2} t_{52} & q t_{42} & q^{1 / 2} t_{32} & t_{22} & q^{-1} t_{12} \\
q^{3} t_{51} & q^{2} t_{41} & q^{3 / 2} t_{31} & q t_{21} & t_{11}
\end{array}\right),
\end{aligned}
$$

where the operation $\dot{\otimes}$ between two matrices $A$ and $B$ is defined as

$$
(A \dot{\otimes} B)_{i j}=A_{i k} \otimes B_{k j} .
$$

To define differential calculus on the non-commutative algebra generated by the coordinates $\mathbf{x}\left(=\left\{x^{i}\right\}, i=-2,-1,0,+1,+2\right)$, we write down the $\hat{\mathcal{R}}(\equiv \mathcal{R} P$, and $P$ a permutation operator $P: \quad A \otimes B=B \otimes A)$ matrix explicitly, 


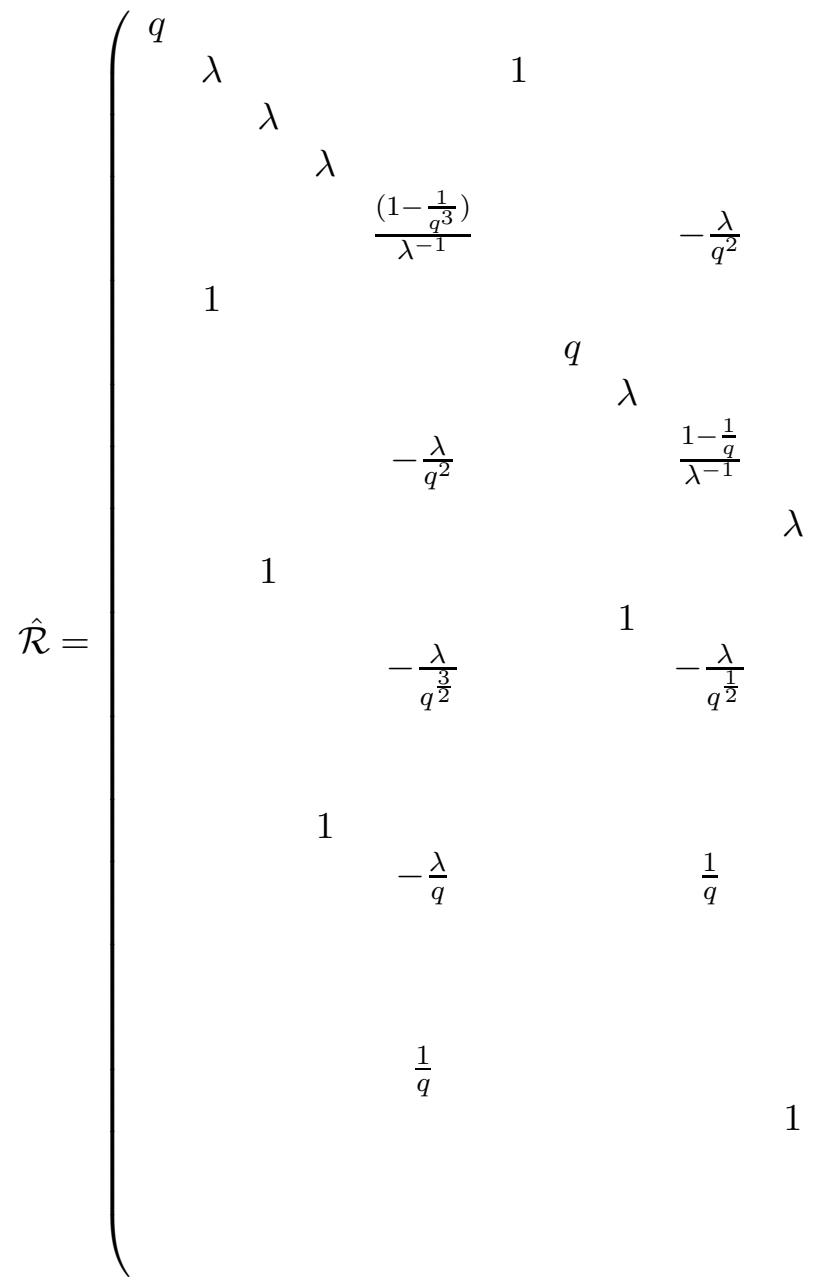

1

1

$-\frac{\lambda}{q^{\frac{3}{2}}}$

$-\frac{\lambda}{q}$

$\frac{1}{q}$

1

$-\frac{\lambda}{q^{\frac{1}{2}}} \quad \frac{1}{q}$

1

1

$\lambda$

$\lambda$

1

1

$q$

1

The $\hat{\mathcal{R}}$ matrix is semisimple and has the spectral resolution

$$
\begin{aligned}
& \hat{\mathcal{R}}=q \mathcal{P}_{S}-q^{-1} \mathcal{P}_{A}+q^{-4} \mathcal{P}_{1} \\
& \hat{\mathcal{R}}^{-1}=q^{-1} \mathcal{P}_{S}-q \mathcal{P}_{A}+q^{4} \mathcal{P}_{1}
\end{aligned}
$$

where $\mathcal{P}_{S}, \mathcal{P}_{A}$ and $\mathcal{P}_{1}$ are projection operators which act on the tensor product $\mathbf{x} \otimes \mathbf{x}$ of the fundamental representation $\mathbf{x}$, and project into the symmetric, antisymmetric and singlet irreducible representations with dimension 14, 10 and 1, respectively. It is convenient to give a concrete representation of the projector,

$$
\begin{aligned}
& \mathcal{P}_{1}=\frac{1-q^{2}}{\left(1-q^{5}\right)\left(1+q^{3}\right)}\left(C^{-1}\right)^{i j} C_{k l} e_{i}^{k} \otimes e_{j}^{l}, \\
& \mathcal{P}_{A}=\frac{1}{q+q^{-1}}\left(-\hat{\mathcal{R}}+q-\left(q-q^{-4}\right) \mathcal{P}_{1}\right) \\
& \mathcal{P}_{S}=\frac{1}{q+q^{-1}}\left(\hat{\mathcal{R}}+q^{-1}-\left(q^{-1}+q^{-4}\right) \mathcal{P}_{1}\right) .
\end{aligned}
$$


Analogous to the commutative case, we define the commutation relations of the quantum orthogonal space by requiring the vanishing of their antisymmetric products. Here the quantum antisymmetric products is given by the projector $\mathcal{P}_{A}$,

$$
\mathcal{P}_{A}(\mathbf{x} \otimes \mathbf{x})=0
$$

In components, the commutation relations among coordinates $x^{i}$ is

$$
\begin{aligned}
& x^{i} x^{j}=q x^{j} x^{i}, \quad \text { for } i<j \text { and } i \neq-j, \\
& q x^{+2} x^{-2}-q^{-1} x^{-2} x^{+2}=\frac{q^{1 / 2}-q^{-1 / 2}}{q-1+q^{-1}} \frac{1}{a^{2}}, \\
& q x^{+1} x^{-1}-q^{-1} x^{-1} x^{+1}=\left(1-q^{2}\right) x^{+2} x^{-2}+q \frac{q^{1 / 2}-q^{-1 / 2}}{q-1+q^{-1}} \frac{1}{a^{2}} .
\end{aligned}
$$

Here we have used the constraint that the quantum length $\left(L \propto \mathbf{x}^{t} C \mathbf{x}=1 / a^{2}\right)$ is invariant under quantum orthogonal group transformations.

The exterior derivative $d[10]$ is an operator which gives the mapping from the coordinates to the differentials

$$
d: \quad x^{i} \longrightarrow d x^{i}
$$

It satisfies two properties

$$
d^{2}=0, \quad d(f g)=(d f) g+(-1)^{f} f(d g) .
$$

where $f$ and $g$ are $p$-forms and $(-1)^{f}$ is $-1(+1)$ if $f$ is odd(even) element. The exterior derivative $d$ is invariant under the quantum group transformation and the differential $d x^{i}$ transforms in the same way as the coordinate $x^{i}$ under the quantum group transformation.

The derivative $\partial_{i}$ can be introduced by

$$
d=d x^{i} \partial_{i}=C_{i j} d x^{i} \partial^{j}
$$

For the differential calculus on the quantum orthogonal space [1], the following relations are satisfied 


$$
\begin{aligned}
& x^{i} d x^{j}=q \hat{R}_{k l}^{i j} d x^{k} x^{l}, \\
& \mathcal{P}_{S}(d \mathbf{x} \wedge d \mathbf{x})=0, \quad \mathcal{P}_{1}(d \mathbf{x} \wedge d \mathbf{x})=0, \\
& \partial^{i} x^{j}=\left(C^{-1}\right)^{i j}+q\left(\hat{\mathcal{R}}^{-1}\right)_{k l}^{i j} x^{k} \partial^{l}, \\
& \mathcal{P}_{A k l}{ }^{i j} \partial_{j} \partial_{i}=0, \\
& \partial^{i} d x^{j}=q^{-1} \hat{\mathcal{R}}_{k l}^{i j} d x^{k} \partial^{l}, \\
& \partial^{i} d=q^{-2} d \partial^{i}-\left(q^{-2}-q^{3}\right) \frac{1-q^{2}}{\left(1-q^{5}\right)\left(1+q^{-3}\right)} d x^{i} C_{j k} \partial^{j} \partial^{k} .
\end{aligned}
$$

\section{Realization of quantum algebra on quantum space}

An action of an algebra $A$ on a space $V$ is a bilinear map,

$$
\mathcal{A}: \quad A \otimes V \longrightarrow V, \quad p \otimes x \longrightarrow p \cdot x
$$

such that

$$
(p q) \cdot x=p \cdot(q \cdot x), \quad 1 \cdot x=1
$$

We call $V$ an $A$-module. In the same sense as comultiplication is the dual to multiplication, coactions are dual to actions. The coaction of a coalgebra $B$ on a space $V$ is a linear map

$$
\omega_{B}: \quad V \longrightarrow B \otimes V
$$

such that

$$
\left(\omega_{B} \otimes \mathrm{id}\right) \omega_{B}=\left(\mathrm{id} \otimes \omega_{B}\right) \omega_{B}, \quad(\mathrm{id} \otimes \epsilon) \omega_{B}=\text { id } .
$$

The coalgebra is referred as a co-module.

We know that the quantum group co-acts on quantum space and is a co-module. From the above general discussion, we conclude that, as a dual Hopf algebra of the quantum group, the quantum algebra acts on the quantum space. Thus, analogue to the classical case, a realization of quantum algebra on the quantum space should be

exist. And when the deformation parameter $q \rightarrow 1$, familiar results on Lie algebra should be recovered. Following these guidances, we present a realization of quantum algebra $U_{q}(s o(5))$ on the quantum orthogonal space.

For convenient, we introduce the dilatation operator $S_{m}(m \leq 2)$ as 


$$
\begin{aligned}
& S_{m}=1+q \lambda E_{m}+q^{2 m+1} \lambda^{2} L_{m} \Delta_{m} \\
& E_{m}=\sum_{j=-m}^{m} x^{j} \partial_{j} \\
& \Delta_{m}=\sum_{j=1}^{m} q^{\rho_{j}} \partial_{j} \partial_{-j}+\frac{q}{1+q} \partial_{0} \partial_{0}, \\
& L_{m}=\sum_{i=1}^{m} q^{\rho_{i}} x^{-i} x^{i}+\frac{q}{1+q} x^{0} x^{0} .
\end{aligned}
$$

The dilatation operator satisfies

$$
S_{m} x^{k}=q^{2} x^{k} S_{m}, \quad S_{m} \partial_{k}=q^{-2} \partial_{k} S_{m}, \quad \text { for }|k| \leq m
$$

Using the notations

$$
\begin{aligned}
& y^{-1}=x^{-1}+q^{3 / 2} \lambda L_{1} \partial_{+1}, \\
& \delta_{-1}=\partial_{-1}+q^{3 / 2} \lambda \Delta_{1} x^{+1} \\
& y^{-2}=x^{-2}+q^{5 / 2} \lambda L_{2} \partial_{+2}, \\
& \delta_{-2}=\partial_{-2}+q^{5 / 2} \lambda \Delta_{2} x^{+2}
\end{aligned}
$$

we can construct a set of independent basis on the quantum orthogonal space

$$
\begin{array}{ll}
\mathcal{X}^{-2}=S_{2}^{-1 / 2} \mu_{+2}^{-1 / 2} y^{-2}, & \mathcal{D}_{-2}=q^{-1} S_{2}^{-1 / 2} \mu_{+2}^{-1 / 2} \delta_{-2}, \\
\mathcal{X}^{-1}=\mu_{+2}^{-1 / 2} S_{1}^{-1 / 2} \mu_{+1}^{-1 / 2} y^{-1}, & \mathcal{D}_{-1}=\mu_{+2}^{-1 / 2} q^{-1} S_{1}^{-1 / 2} \mu_{+1}^{-1 / 2} \delta_{-1} \\
\mathcal{X}^{0}=\mu_{+2}^{-1 / 2} \mu_{+1}^{-1 / 2} x^{0}, & \mathcal{D}_{0}=\mu_{+2}^{-1 / 2} \mu_{+1}^{-1 / 2} \partial_{0} \\
\mathcal{X}^{+1}=\mu_{+2}^{-1 / 2} x^{+1}, & \mathcal{D}_{+1}=\mu_{+2}^{-1 / 2} \partial_{+1} \\
\mathcal{X}^{2}=x^{2}, & \mathcal{D}_{2}=\partial_{2}
\end{array}
$$

where $\left(\mu_{ \pm i}\right)^{ \pm 1}=\mathcal{D}_{ \pm i} \mathcal{X}^{ \pm i}-\mathcal{X}^{ \pm i} \mathcal{D}_{ \pm i}$ and $\mu_{0}^{1 / 2}=\mathcal{D}_{0} \mathcal{X}^{0}-\mathcal{X}^{0} \mathcal{D}_{0}$

We note that the $\mu_{i}$ 's satisfy simple commutation relations with the independent basis $\mathcal{X}$ and $\mathcal{D}$

$\left[\mu_{i}, \mu_{j}\right]=0, \quad \mu_{i} \mathcal{X}^{j}=\mathcal{X}^{j} \mu_{i} \cdot\left\{\begin{array}{l}q^{2}, \quad \text { for } i=j \\ 1, \quad \text { for } i \neq j\end{array}, \quad \mu_{i} \mathcal{D}^{j}=\mathcal{D}^{j} \mu_{i} \cdot\left\{\begin{array}{l}q^{-2}, \quad \text { for } i=j \\ 1, \quad \text { for } i \neq j\end{array}\right.\right.$

For the independent basis of coordinates and derivatives on the quantum space [12] [13], it is not difficult to show that 


$$
\begin{aligned}
& \mathcal{D}_{-2} \mathcal{X}^{-2}=1+q^{-2} \mathcal{X}^{-2} \mathcal{D}_{-2}, \\
& \mathcal{D}_{-1} \mathcal{X}^{-1}=1+q^{-2} \mathcal{X}^{-1} \mathcal{D}_{-1}, \\
& \mathcal{D}_{0} \mathcal{X}^{0}=1+q \mathcal{X}^{0} \mathcal{D}_{0}, \\
& \mathcal{D}_{+1} \mathcal{X}^{+1}=1+q^{2} \mathcal{X}^{+1} \mathcal{D}_{+1}, \\
& \mathcal{D}_{+2} \mathcal{X}^{+2}=1+q^{2} \mathcal{X}^{+2} \mathcal{D}_{+2}, \\
& {\left[\mathcal{D}_{i}, \mathcal{D}_{j}\right]=0, \quad\left[\mathcal{X}^{i}, \mathcal{X}^{j}\right]=0,} \\
& \mathcal{D}_{i} \mathcal{X}^{j}=\mathcal{X}^{j} \mathcal{D}_{i}, \quad \text { for } i \neq j .
\end{aligned}
$$

We know the Cartan matrix for its rank two Lie algebra $B_{2}$ is

$$
A_{i j}=\left(\begin{array}{cc}
2 & -1 \\
-2 & 2
\end{array}\right), \quad\left\langle\alpha_{i}, \alpha_{j}\right\rangle=\left(\begin{array}{cc}
2 & -1 \\
-1 & 1
\end{array}\right) .
$$

So that $d_{1}=1, d_{2}=1 / 2$.

The quantum universal enveloping algebra 14 U $U_{q}(s o(5))$ is

$$
\begin{aligned}
& {\left[\tilde{H}_{i}, \tilde{H}_{j}\right]=0, \quad i, j=1,2,} \\
& {\left[\tilde{H}_{i}, \tilde{X}_{j}^{ \pm}\right]= \pm a_{i j} \tilde{X}_{j}^{ \pm},} \\
& {\left[\tilde{X}_{i}^{+}, \tilde{X}_{j}^{-}\right]=\delta_{i j} \frac{q_{i}^{\tilde{H}_{i}}-q_{i}^{-\tilde{H}_{i}}}{q_{i}-q_{i}^{-1}}, \quad q_{i} \equiv q^{d_{i}},} \\
& \sum_{m=0}^{1-a_{i j}}\left[\begin{array}{c}
1-a_{i j} \\
m
\end{array}\right]_{q_{i}}\left(\tilde{X}_{i}^{ \pm}\right)^{m} \tilde{X}_{j}^{ \pm}\left(\tilde{X}_{i}^{ \pm}\right)^{1-a_{i j}-m}=0, \quad i \neq 0,
\end{aligned}
$$

where $[m]_{q}=\frac{q^{m}-q^{-m}}{q-q^{-1}}$, and $\left[\begin{array}{c}m \\ n\end{array}\right]_{q}=\frac{[m]_{q} ![m-n]_{q} !}{[n]_{q} !}$.

Redefine $H_{i}=d_{i} \tilde{H}_{i}, X_{i}^{ \pm}=\sqrt{\left[d_{i}\right]_{q}} \tilde{X}_{i}^{ \pm}$, we have

$$
\begin{aligned}
& {\left[H_{1}, H_{2}\right]=0} \\
& {\left[H_{1}, X_{1}^{ \pm}\right]= \pm 2 X_{1}^{ \pm}, \quad\left[H_{1}, X_{2}^{ \pm}\right]=\mp X_{2}^{ \pm}} \\
& {\left[H_{2}, X_{1}^{ \pm}\right]=\mp X_{1}^{ \pm}, \quad\left[H_{2}, X_{2}^{ \pm}\right]= \pm X_{2}^{ \pm}} \\
& {\left[X_{1}^{+}, X_{1}^{-}\right]=\frac{q^{H_{1}}-q^{-H_{1}}}{q-q^{-1}}} \\
& {\left[X_{2}^{+}, X_{2}^{-}\right]=\frac{q^{H_{2}}-q^{-H_{2}}}{q-q^{-1}}} \\
& X_{2}^{ \pm}\left(X_{1}^{ \pm}\right)^{2}-[2] X_{1}^{ \pm} X_{2}^{ \pm} X_{1}^{ \pm}+\left(X_{1}^{ \pm}\right)^{2} X_{2}^{ \pm}=0, \\
& X_{1}^{ \pm}\left(X_{2}^{ \pm}\right)^{3}-[3]_{\sqrt{q}} X_{2}^{ \pm} X_{1}^{ \pm}\left(X_{2}^{ \pm}\right)^{2}+[3]_{\sqrt{q}}\left(X_{2}^{ \pm}\right)^{2} X_{1}^{ \pm} X_{2}^{ \pm}-\left(X_{2}^{ \pm}\right)^{3} X_{1}^{ \pm}=0
\end{aligned}
$$

The Hopf algebra structure of the quantum universal enveloping algebra $U_{q}(s o(5))$ is of the form 


$$
\begin{aligned}
& \Delta\left(H_{i}\right)=H_{i} \otimes 1+1 \otimes H_{i}, \\
& \Delta\left(X_{i}^{ \pm}\right)=X_{i}^{ \pm} \otimes q^{H_{i} / 2}+q^{-H_{i} / 2} \otimes X_{i}^{ \pm}, \\
& \epsilon\left(H_{i}\right)=0=\epsilon\left(X_{i}^{ \pm}\right), \\
& S\left(H_{i}\right)=-H_{i}, \quad S\left(X_{i}^{ \pm}\right)=-q^{\mp d_{i}} X_{i}^{ \pm} .
\end{aligned}
$$

In terms of the set of independent basis $\mathcal{X}^{i}, \mathcal{D}_{i}$ on the quantum space, we write down a realization of the quantum universal enveloping algebra $U_{q}(s o(5))$ in fundamental representation,

$$
\begin{aligned}
q^{H_{1}} & =\left(\frac{\mu_{-2} \mu_{+1}}{\mu_{-1} \mu_{+2}}\right)^{1 / 2}, \\
X_{1}^{-} & =\sqrt{q^{-1} \lambda}\left(\frac{\mu_{-1} \mu_{+2}}{\mu_{-2} \mu_{+1}}\right)^{1 / 4} \mu_{+2}^{-1 / 2}\left(\left(\mu_{-2} \mu_{+1}\right)^{1 / 2} \mathcal{X}^{-1} \mathcal{D}_{-2}-q \mathcal{X}^{+2} \mathcal{D}_{+1}\right) \\
X_{1}^{+} & =\sqrt{q^{-1} \lambda}\left(\frac{\mu_{-1} \mu_{+2}}{\mu_{-2} \mu_{+1}}\right)^{1 / 4} \mu_{+2}^{-1 / 2}\left(\left(\mu_{-2} \mu_{+1}\right)^{1 / 2} \mathcal{X}^{-2} \mathcal{D}_{-1}-q \mathcal{X}^{+1} \mathcal{D}_{+2}\right) \\
q^{H_{2}} & =\left(\frac{\mu_{-1}}{\mu_{+1}}\right)^{1 / 2} \\
X_{2}^{-} & =\sqrt{q^{1 / 2} \lambda}\left(\mu_{-1} \mu_{+1}\right)^{-1 / 4}\left(q^{-3 / 2} \mu_{-1}^{1 / 2} \mathcal{X}^{0} \mathcal{D}_{-1}-\mathcal{X}^{+1} \mathcal{D}_{0}\right) \\
X_{2}^{+} & =\sqrt{q^{1 / 2} \lambda}\left(\mu_{-1} \mu_{+1}\right)^{-1 / 4}\left(q^{1 / 2} \mu_{-1}^{1 / 2} \mathcal{X}^{-1} \mathcal{D}_{0}-\mathcal{X}^{0} \mathcal{D}_{+1}\right)
\end{aligned}
$$

Therefore, we indeed have a natural action of the quantum algebra $U_{q}(s o(5))$ on the quantum orthogonal space. It may be referred as the quantum counterpart of the Lie algebra realized on Euclidean space. In fact, at the case of $q=1$, this realization recovered a ordinary realization of Lie algebra. This is helpful for clearly understanding of meanings of quantum group and quantum algebra.

\section{Quantum AdS group and quantum AdS space}

To study quantum AdS group, real forms and *-conjugations of quantum orthogonal group should be introduced. A $*$-structure on a Hopf algebra $A$ is an algebra antiautomorphism $(\eta a b)^{*}=\bar{\eta} b^{*} a^{*}(\forall a, b \in A, \forall \eta \in C)$, coalgebra automorphism $\Delta \cdot *=$ $(* \otimes *) \cdot \Delta, \epsilon \cdot *=\epsilon$ and involution $*^{2}=1$.

Following FRT [9], on quantum orthogonal groups conjugations can be defined. The first type is trivially as $T^{\times}=T$. Compatibility with the Yang-Baxter equation requires $\overline{\mathcal{R}}=\mathcal{R}^{-1}$ and $\bar{C} C^{t}=1$. This occurs only for $|q|=1$. Then the $C T T$ relations are

invariant under the *-conjugation. As we know the quantum orthogonal group coacts on the quantum orthogonal space, and may induce an associated conjugation 
on the quantum space as well. More precisely a conjugation on the quantum space is compatible with a conjugation on its quantum symmetry group if the coaction $\omega\left(x^{a}\right)=$ $T_{b}^{a} \otimes x^{b}$ satisfies $\omega\left(\mathbf{x}^{\times}\right)=T^{\times} \otimes \mathbf{x}^{\times} \equiv \delta^{\times}(\mathbf{x})$. The unique associated quantum space conjugation here is $\left(x^{a}\right)^{\times}=x^{a}$. Clearly, we can not get the desired quantum AdS space by this kind of conjugation on quantum orthogonal space. Further structure should be added to the conjugation to arrive at the quantum AdS group and quantum AdS space. For the aim, we introduce another operation on the quantum orthogonal group as

$$
T^{\dagger}=\mathcal{D} T \mathcal{D}^{-1}
$$

where the matrix $\mathcal{D}$ is of the form

$$
\mathcal{D}=\left(\begin{array}{lllll}
1 & & & & \\
& 1 & & & \\
& & -1 & & \\
& & & 1 & \\
& & & & 1
\end{array}\right) .
$$

It is easy to check that the $\mathcal{D}$ matrix is a special element of the quantum orthogonal group[15], i.e.,

$$
\begin{aligned}
& \mathcal{R}_{12} \mathcal{D}_{1} \mathcal{D}_{2}=\mathcal{D}_{2} \mathcal{D}_{1} \mathcal{R}_{12}, \\
& \mathcal{D}^{t} C \mathcal{D}=C, \quad \mathcal{D} C \mathcal{D}^{t}=C
\end{aligned}
$$

Thus the $\dagger$ operation is compatible with the Hopf algebra structure of the quantum orthogonal group,

$$
\begin{aligned}
& \mathcal{R}_{12} T_{1}^{\dagger} T_{2}^{\dagger}=T_{2}^{\dagger} T_{1}^{\dagger} \mathcal{R}_{12}, \\
& \left(T^{\dagger}\right)^{t} C T^{\dagger}=C, \quad T^{\dagger} C\left(T^{\dagger}\right)^{t}=C, \\
& \Delta\left(T^{\dagger}\right)=\mathcal{D} \Delta(T) \mathcal{D}^{-1}=T^{\dagger} \otimes T^{\dagger}, \\
& \epsilon\left(T^{\dagger}\right)=\epsilon(T), \quad S\left(T^{\dagger}\right)=[S(T)]^{\dagger} .
\end{aligned}
$$

We finally obtain the desired AdS quantum group by using the combined operation of the $\times$ and $\dagger$, i.e., the conjugation $T^{*} \equiv T^{\times \dagger}=\mathcal{D} T \mathcal{D}^{-1}$. The induced conjugation on the quantum space is $\mathbf{x}^{*} \equiv \mathbf{x}^{\times \dagger}=\mathcal{D} \mathbf{x}$. To show that the conjugation really gives the quantum AdS group and quantum AdS space, we should find a linear transformation $\mathbf{x} \longrightarrow \mathbf{x}^{\prime}=M \mathbf{x}, T \longrightarrow T^{\prime}=M T M^{-1}$ such that the new coordinates $\mathbf{x}^{\prime}$ and $T^{\prime}$ are real and the new metric $C^{\prime}=\left(M^{-1}\right)^{t} C M^{-1}$ diagonal in the $q \longrightarrow 1$ limit, $\left.C^{\prime}\right|_{q=1}=$ 
$\operatorname{diag}(1,-1,-1,-1,1)$. Here the $M$ matrix is given by

$$
M=\frac{1}{\sqrt{2}}\left(\begin{array}{ccccc}
1 & & & & 1 \\
-1 & & & & 1 \\
& & i \sqrt{2} & & \\
& -1 & 1 & \\
& 1 & & 1
\end{array}\right) .
$$

The second conjugation of the quantum orthogonal group given by FRT [9] is realized via the metric, i.e., $T^{\star}=C^{t} T C^{t}$. The condition on the quantum $\mathcal{R}$ matrix is $\overline{\mathcal{R}}=\mathcal{R}$, which happens for $q \in R$. Again the $C T T$ relations are invariant under such a conjugation operation. The corresponding real form is $S O_{q}(5 ; R)$. The induced conjugation on the quantum space is $\mathbf{x}^{\star}=C^{t} \mathbf{x}$. Again, we can not get the desired quantum AdS group and quantum AdS space by this kind of conjugation along. To obtain quantum AdS group, we introduce the operation $\ddagger$ on the quantum orthogonal group $S O_{q}(5)$ as

$$
T^{\ddagger}=\mathcal{N} T \mathcal{N}^{-1},
$$

where $\mathcal{N}$ matrix is of the form

$$
\mathcal{N}=\left(\begin{array}{ccccc}
1 & & & & \\
& -1 & & & \\
& & -1 & & \\
& & & -1 & \\
& & & & 1
\end{array}\right) .
$$

The $\mathcal{N}$ matrix is also a special element of the quantum orthogonal group, i.e.,

$$
\begin{aligned}
& \mathcal{R}_{12} \mathcal{N}_{1} \mathcal{N}_{2}=\mathcal{N}_{2} \mathcal{N}_{1} \mathcal{R}_{12} \\
& \mathcal{N}^{t} C \mathcal{N}=C, \quad \mathcal{N} C \mathcal{N}^{t}=C .
\end{aligned}
$$

Thus the $\ddagger$ operation is also compatible with the Hopf algebra structure of quantum group. We obtain the desired AdS quantum group by using the combined operation of the $\star$ and $\ddagger$, i.e., the conjugation $T^{*} \equiv T^{\star \ddagger}=\mathcal{N} C^{t} T C^{t} \mathcal{N}^{-1}$. The induced conjugation on the quantum space is $\mathbf{x}^{*} \equiv \mathbf{x}^{\star \ddagger}=C^{t} \mathcal{N} \mathbf{x}$.

Parallel to the case of $|q|=1$, we prove the combined conjugation really gives the quantum AdS group and quantum AdS space by trying to find a linear transformation 
$\mathbf{x} \longrightarrow \mathbf{x}^{\prime}=N \mathbf{x}, T \longrightarrow T^{\prime}=N T N^{-1}$ such that the new coordinates $\mathbf{x}^{\prime}$ and $T^{\prime}$ are real and the new metric $C^{\prime}=\left(N^{-1}\right)^{t} C N^{-1}$ diagonal in the $q \longrightarrow 1$ limit, $\left.C^{\prime}\right|_{q=1}=$ $\operatorname{diag}(1,-1,-1,-1,1)$. We find a $N$ matrix which satisfies all these requirements of the form

$$
N=\frac{1}{\sqrt{2}}\left(\begin{array}{ccccc}
1 & & & & q^{3 / 2} \\
& -i & & -i q^{1 / 2} & \\
& q^{-1 / 2} & & -1 & \\
i q^{-3 / 2} & & & & -i
\end{array}\right)
$$

\section{Differential Calculus on the quantum AdS space}

To study differential calculus on the quantum AdS space, we should introduce conjugate derivatives. Because there are two different conjugations on the quantum AdS space corresponds to the deformation parameter $q$ being root of unit or real, respectively. We present differential calculus on the quantum AdS space for the two cases separately.

In the case of $q$ being root of unit, we note the conjugation on $x^{i}, \hat{\mathcal{R}}$ and $C$ is given by

$$
x^{i *}=\mathcal{D}_{i j} x^{j}, \quad \overline{\hat{\mathcal{R}}_{i j}^{u v}}=\hat{\mathcal{R}}^{-1}{ }_{j i}^{v u}, \quad \overline{C^{i j}}=C^{j i} .
$$

Because the matrix $\mathcal{D}$ is a special element of the quantum orthogonal group, we can prove that

$$
\hat{\mathcal{R}}^{-1}{ }_{l d}^{i k} \mathcal{D}_{k n} \mathcal{D}_{s l} \mathcal{D}_{i p} \mathcal{D}_{t d}=\hat{\mathcal{R}}^{-1}{ }_{p n}^{s t}
$$

The $\hat{\mathcal{R}}$-matrix has the following symmetry properties

$$
C^{i m} \hat{\mathcal{R}}_{m k}^{j n} C_{n l}=\hat{\mathcal{R}}^{-1}{ }_{k l}^{i j}=C_{k m} \hat{\mathcal{R}}_{l n}^{m i} C^{n j}
$$

and

$$
\hat{\mathcal{R}}_{k l}^{i j}=\hat{\mathcal{R}}_{i j}^{k l}
$$


Using the above relations as well as the conjugation of the definition of derivatives, $\partial_{i} x^{j}=\delta_{i}^{j}+q \hat{\mathcal{R}}_{i l}^{j k} x^{l} \partial_{k}$, we obtain the action of the conjugate derivatives on the quantum AdS space,

$$
\hat{\partial}_{m} x^{s}=\delta_{m}^{s}+q \hat{\mathcal{R}}_{m k}^{s l} x^{k} \hat{\partial}_{l}
$$

where $\hat{\partial}_{m} \equiv-q^{-1} \mathcal{D}_{m k} C_{k v} \partial^{v *}$.

This show that the action of the conjugate derivatives on the quantum AdS space is almost the same with that of the derivatives at the case of $|q|=1$. In fact, we can represent the conjugate derivatives $\partial^{*}$ linearly in terms of the derivatives $\partial$,

$$
\partial_{v}^{*}=-q C_{v k} \mathcal{D}_{k m} \partial_{m}
$$

From a very general consideration[10], we know that there are two types of consistent derivatives $\partial_{i}$ and $\tilde{\partial}_{i}$ satisfy the following relations, respectively,

$$
\begin{aligned}
& \partial_{i} x^{j}=\delta_{i}^{j}+q \hat{\mathcal{R}}_{i l}^{j k} x^{l} \partial_{k}, \\
& \tilde{\partial}_{k} x^{v}=\delta_{k}^{v}+q^{-1} \hat{\mathcal{R}}^{-1}{ }_{k j}{ }^{j i}{ }^{j} \tilde{\partial}_{i} .
\end{aligned}
$$

The first one is just what given in Eq.(9). We show that the second one is related with the conjugate derivatives in the case of $q \in R$.

In the following, we present actions of the conjugate derivatives on the quantum AdS space at the case of $q \in R$. To proceed, we first conjugate the first relation in Eq.(26) and invert it to find an expression for $\partial_{i}^{*} x^{j}$ in terms of $x^{j} \partial_{i}^{*}$ by using

$$
x^{i *}=C_{j i} \mathcal{N}_{j k} x^{k}
$$

and the symmetry properties of the $\hat{\mathcal{R}}$-matrix Eq.(22) and (23). The result is:

$$
-q^{5} \mathcal{N}_{i p} C_{i a} C^{v a} \partial^{v *} x^{s}=q^{4} C_{i a} C_{p \delta} \hat{\mathcal{R}}_{a l}^{p \delta} \mathcal{N}_{s l} \mathcal{N}_{i p}-q^{4} \hat{\mathcal{R}}^{-1}{ }_{l d}^{i k} \mathcal{N}_{k n} \mathcal{N}_{s l} \mathcal{N}_{i p} \mathcal{N}_{t d} x^{n} C_{d b} C^{m b} \mathcal{N}_{d t} \partial^{m *}
$$

Making use of Eq.(20), we know that 


$$
\hat{\mathcal{R}}^{-1}{ }_{l d}^{i k} \mathcal{N}_{k n} \mathcal{N}_{s l} \mathcal{N}_{i p} \mathcal{N}_{t d}=\hat{\mathcal{R}}^{-1}{ }_{p n}^{s t}
$$

Then, we get

$$
\hat{\partial}_{p} x^{s}=\delta_{p}^{s}+q^{-1} \hat{\mathcal{R}}^{-1}{ }_{p n}^{s t} x^{n} \hat{\partial}_{t}
$$

where

$$
\hat{\partial}_{p}=-q^{5} \mathcal{N}_{i p} C_{i a} C^{v a} \partial^{v *}
$$

$\hat{\partial}_{i}$ (or equivalient $\partial_{i}^{*}$ ) can be expressed algebraically in terms of $x^{k}$ and $\partial_{l}$ [16],

$$
\hat{\partial}_{k}=q^{3} S_{2}^{-1}\left[\Delta_{2}, x^{k}\right]
$$

To complete the differential calculus on the quantum AdS space, we give the consistent relations satisfied by $\hat{\partial}$ and $\partial$

$$
\begin{aligned}
& \hat{\partial}_{i} \hat{\partial}_{j}=q^{-1} \hat{\mathcal{R}}_{j i}^{l k} \hat{\partial}_{k} \hat{\partial}_{l}, \\
& \hat{\partial}_{i} \partial_{j}=q \hat{\mathcal{R}}_{j i}^{l k} \partial_{k} \hat{\partial}_{l} .
\end{aligned}
$$

\section{Quantum group via quantum algebra}

It is well known that, in Lie group theory, there is an exponential corresponding between group and algebra. Representations of Lie group are easily deduced from that of Lie algebra. And then quantum field theory is constructed based on representations of Lie algebra. However, in general, there is no such a direct transformation from quantum algebra to quantum group, besides duality between them. Bearing the goal of constructing quantum field theory on noncommutative geometry, we try to set up an explicit relationship [17] between quantum orthogonal group and quantum orthogonal algebra.

It is convenient to introduce the operator $L^{+}$as 


$$
L^{+}=\left(\begin{array}{ccccc}
l_{11}^{+} & l_{12}^{+} & l_{13}^{+} & l_{14}^{+} & l_{15}^{+} \\
& l_{22}^{+} & l_{23}^{+} & l_{24}^{+} & l_{25}^{+} \\
& & l_{33}^{+} & l_{34}^{+} & l_{35}^{+} \\
& & & l_{44}^{+} & l_{45}^{+} \\
& & & & l_{55}^{+}
\end{array}\right)
$$

where

$$
\begin{aligned}
& l_{11}^{+}=q^{H_{1}+H_{2}} \\
& l_{12}^{+}=\lambda q^{-1 / 2} X_{1}^{+} q^{\frac{H_{1}}{2}+H_{2}} \\
& l_{13}^{+}=\lambda q^{-1 / 2}\left(X_{1}^{+} X_{2}^{+}-q^{-1} X_{2}^{+} X_{1}^{+}\right) q^{\frac{H_{1}+H_{2}}{2}}, \\
& l_{14}^{+}=\lambda q^{-5 / 2}\left(-q X_{1}^{+}\left(X_{2}^{+}\right)^{2}+(q+1) X_{2}^{+} X_{1}^{+} X_{2}^{+}-\left(X_{2}^{+}\right)^{2} X_{1}^{+}\right) q^{H_{1} / 2} \\
& l_{15}^{+}=\frac{\lambda^{2} q^{-2}}{1+q}\left(\left(X_{1}^{+}\right)^{2}\left(X_{2}^{+}\right)^{2}-\left(q+1+q^{-1}\right) X_{1}^{+} X_{2}^{+} X_{1}^{+} X_{2}^{+}+\left(q+1+q^{-1}\right) X_{1}^{+}\left(X_{2}^{+}\right)^{2} X_{1}^{+}\right. \\
& \left.\quad \quad-\left(q+1+q^{-1}\right) X_{2}^{+} X_{1}^{+} X_{2}^{+} X_{1}^{+}+X_{2}^{+}\left(X_{1}^{+}\right)^{2} X_{2}^{+}+\left(X_{2}^{+}\right)^{2}\left(X_{1}^{+}\right)^{2}\right) \\
& l_{22}^{+}=q^{H_{2}}, \quad \\
& l_{23}^{+}=\lambda q^{-1 / 2} X_{2}^{+} q^{H_{2} / 2} \\
& l_{24}^{+}=-\frac{\lambda^{2}}{q(1+q)}\left(X_{2}^{+}\right)^{2}, \\
& l_{25}^{+}=\lambda q^{-5 / 2}\left(X_{1}^{+}\left(X_{2}^{+}\right)^{2}-(1+q) X_{2}^{+} X_{1}^{+} X_{2}^{+}+q\left(X_{2}^{+}\right)^{2} X_{1}^{+}\right) q^{-H_{1} / 2} \\
& l_{33}^{+}=1 \\
& l_{34}^{+}=-\lambda q^{-1} X_{2}^{+} q^{-H_{2} / 2} \\
& l_{35}^{+}=\lambda q^{-1}\left(-q^{-1} X_{1}^{+} X_{2}^{+}+X_{2}^{+} X_{1}^{+}\right) q^{-\frac{H_{1}+H_{2}}{2}} \\
& l_{44}^{+}=q^{-H_{2}}, \\
& l_{45}^{+}=-\lambda q^{-1 / 2} X_{1}^{+} q^{-\left(\frac{H_{1}}{2}+H_{2}\right)} \\
& l_{55}^{+}=q^{-\left(H_{1}+H_{2}\right)} .
\end{aligned}
$$

In the same manner, we introduce another operator $L^{-}$as

$$
L^{-}=\left(\begin{array}{ccccc}
l_{11}^{-} & & & & \\
l_{21}^{-} & l_{22}^{-} & & & \\
l_{31}^{-} & l_{32}^{-} & l_{33}^{-} & & \\
l_{41}^{-} & l_{42}^{-} & l_{43}^{-} & l_{44}^{-} & \\
l_{51}^{-} & l_{52}^{-} & l_{53}^{-} & l_{54}^{-} & l_{55}^{-}
\end{array}\right),
$$


where

$$
\begin{aligned}
& l_{11}^{-}=q^{-\left(H_{1}+H_{2}\right)} \\
& l_{21}^{-}=-\lambda q^{1 / 2} X_{1}^{-} q^{-\left(\frac{H_{1}}{2}+H_{2}\right)} \\
& l_{31}^{-}=\lambda q^{3 / 2}\left(X_{1}^{-} X_{2}^{-}-q^{-1} X_{2}^{-} X_{1}^{-}\right) q^{-\frac{H_{1}+H_{2}}{2}}, \\
& l_{41}^{-}=\lambda q^{1 / 2}\left(q X_{1}^{-}\left(X_{2}^{-}\right)^{2}-(q+1) X_{2}^{-} X_{1}^{-} X_{2}^{-}+\left(X_{2}^{-}\right)^{2} X_{1}^{-}\right) q^{-H_{1} / 2} \\
& l_{51}^{-}=\frac{\lambda^{2} q^{2}}{1+q}\left(\left(X_{1}^{-}\right)^{2}\left(X_{2}^{-}\right)^{2}-\left(q+1+q^{-1}\right) X_{1}^{-} X_{2}^{-} X_{1}^{-} X_{2}^{-}+\left(q+1+q^{-1}\right) X_{1}^{-}\left(X_{2}^{-}\right)^{2} X_{1}^{-}\right. \\
& \left.\quad \quad-\left(q+1+q^{-1}\right) X_{2}^{-} X_{1}^{-} X_{2}^{-} X_{1}^{-}+X_{2}^{-}\left(X_{1}^{-}\right)^{2} X_{2}^{-}+\left(X_{2}^{-}\right)^{2}\left(X_{1}^{-}\right)^{2}\right) \\
& l_{22}^{-}=q^{-H_{2}}, \\
& l_{32}^{-}=-\lambda q^{1 / 2} X_{2}^{-} q^{-H_{2} / 2} \\
& l_{42}^{-}=-\frac{\lambda^{2} q}{1+q}\left(X_{2}^{-}\right)^{2} \\
& l_{52}^{-}=\lambda q^{1 / 2}\left(-X_{1}^{-}\left(X_{2}^{-}\right)^{2}+(1+q) X_{2}^{-} X_{1}^{-} X_{2}^{-}-q\left(X_{2}^{-}\right)^{2} X_{1}^{-}\right) q^{H_{1} / 2} \\
& l_{33}^{-}=1 \\
& l_{43}^{-}=\lambda X_{2}^{-} q^{H_{2} / 2} \\
& l_{53}^{-}=\lambda q\left(-q^{-1} X_{1}^{-} X_{2}^{-}+X_{2}^{-} X_{1}^{-}\right) q^{\frac{H_{1}+H_{2}}{2}} \\
& l_{44}^{-}=q^{H_{2}} \\
& l_{54}^{-}=\lambda q^{1 / 2} X_{1}^{-} q^{\frac{H_{1}}{2}+H_{2}} \\
& l_{55}^{-}=q^{H_{1}+H_{2}} .
\end{aligned}
$$

By making use of the operators $L^{ \pm}$, we can write the quantum universal enveloping algebra $U_{q}(s o(5))$ into a compact form

$$
\begin{aligned}
& \mathcal{R}_{12} L_{1}^{ \pm} L_{2}^{ \pm}=L_{2}^{ \pm} L_{1}^{ \pm} \mathcal{R}_{12} \text {, } \\
& \mathcal{R}_{12} L_{1}^{-} L_{2}^{+}=L_{2}^{+} L_{1}^{-} \mathcal{R}_{12} \text {, } \\
& \Delta\left(L^{ \pm}\right)=L^{ \pm} \dot{\otimes} L^{ \pm}, \quad \epsilon\left(L^{ \pm}\right)=1 ， \\
& S\left(L^{+}\right)=\left(\begin{array}{ccccc}
l_{55}^{+} & q^{-1} l_{45}^{+} & q^{-3 / 2} l_{35}^{+} & q^{-2} l_{25}^{+} & q^{-3} l_{15}^{+} \\
& l_{44}^{+} & q^{-1 / 2} l_{34}^{+} & q^{-1} l_{24}^{+} & q^{-2} l_{14}^{+} \\
& & l_{33}^{+} & q^{-1 / 2} l_{23}^{+} & q^{-3 / 2} l_{13}^{+} \\
& & & l_{22}^{+} & q^{-1} l_{21}^{+} \\
& & & & \\
& & & &
\end{array}\right. \\
& S\left(L^{-}\right)=\left(\begin{array}{ccccc}
l_{55}^{-} & & & & \\
q l_{54}^{-} & l_{44}^{-} & & & \\
q^{3 / 2} l_{53}^{-} & q^{1 / 2} l_{43}^{-} & l_{33}^{-} & & \\
q^{2} l_{52}^{-} & q l_{42}^{-} & q^{1 / 2} l_{32}^{-} & l_{22}^{-} & \\
q^{3} l_{51}^{-} & q^{2} l_{41}^{-} & q^{3 / 2} l_{31}^{-} & q l_{21}^{-} & l_{11}^{-}
\end{array}\right) .
\end{aligned}
$$

As well known, in the classical case, many important properties of Lie group are conveniently studied using corresponding Lie algebra and vise verse. This is based on the exponential relation of Lie group and Lie algebra. However, in the quantum group theory, there is no such a counterpart of exponential has been found. All we know is 
the duality between quantum group and quantum algebra. We give here an explicit relation between elements of quantum group and generators of the quantum universal enveloping algebra,

$$
T=L^{+} \dot{\otimes} L^{-}, \quad \text { or } \quad T=L^{-} \dot{\otimes} L^{+}
$$

Then elements of the $T$ matrix are expressed as

$$
\begin{aligned}
& t_{11}=l_{11}^{+} \otimes l_{11}^{-}+l_{12}^{+} \otimes l_{21}^{-}+l_{13}^{+} \otimes l_{31}^{-}+l_{14}^{+} \otimes l_{41}^{-}+l_{15}^{+} \otimes l_{51}^{-}, \\
& t_{12}=l_{12}^{+} \otimes l_{22}^{-}+l_{13}^{+} \otimes l_{32}^{-}+l_{14}^{+} \otimes l_{42}^{-}+l_{15}^{+} \otimes l_{52}^{-}, \\
& t_{13}=l_{13}^{+} \otimes l_{33}^{-}+l_{14}^{+} \otimes l_{43}^{-}+l_{15}^{+} \otimes l_{53}^{-}, \\
& t_{14}=l_{14}^{+} \otimes l_{44}^{-}+l_{15}^{+} \otimes l_{54}^{-} \\
& t_{15}=l_{15}^{+} \otimes l_{55}^{-} \\
& t_{21}=l_{22}^{+} \otimes l_{21}^{-}+l_{23}^{+} \otimes l_{31}^{-}+l_{24}^{+} \otimes l_{41}^{-}+l_{25}^{+} \otimes l_{51}^{-}, \\
& t_{22}=l_{22}^{+} \otimes l_{22}^{-}+l_{23}^{+} \otimes l_{32}^{-}+l_{24}^{+} \otimes l_{42}^{-}+l_{25}^{+} \otimes l_{52}^{-}, \\
& t_{23}=l_{23}^{+} \otimes l_{33}^{-}+l_{24}^{+} \otimes l_{43}^{-}+l_{25}^{+} \otimes l_{53}^{-}, \\
& t_{24}=l_{24}^{+} \otimes l_{44}^{-}+l_{25}^{+} \otimes l_{54}^{-}, \\
& t_{25}=l_{25}^{+} \otimes l_{55}^{-}, \\
& t_{31}=l_{33}^{+} \otimes l_{31}^{-}+l_{34}^{+} \otimes l_{41}^{-}+l_{35}^{+} \otimes l_{51}^{-}, \\
& t_{32}=l_{33}^{+} \otimes l_{32}^{-}+l_{34}^{+} \otimes l_{42}^{-}+l_{35}^{+} \otimes l_{52}^{-}, \\
& t_{33}=l_{33}^{+} \otimes l_{33}^{-}+l_{34}^{+} \otimes l_{43}^{-}+l_{35}^{+} \otimes l_{53}^{-}, \\
& t_{34}=l_{34}^{+} \otimes l_{44}^{-}+l_{35}^{+} \otimes l_{54}^{-} \\
& t_{35}=l_{35}^{+} \otimes l_{55}^{-} \\
& t_{41}=l_{44}^{+} \otimes l_{41}^{-}+l_{45}^{+} \otimes l_{51}^{-} \\
& t_{42}=l_{44}^{+} \otimes l_{42}^{-}+l_{45}^{+} \otimes l_{52}^{-} \\
& t_{43}=l_{44}^{+} \otimes l_{43}^{-}+l_{45}^{+} \otimes l_{53}^{-} \\
& t_{44}=l_{44}^{+} \otimes l_{44}^{-}+l_{45}^{+} \otimes l_{54}^{-} \\
& t_{45}=l_{45}^{+} \otimes l_{55}^{-} \\
& t_{51}=l_{55}^{+} \otimes l_{51}^{-} \\
& t_{52}=l_{55}^{+} \otimes l_{52}^{-} \\
& t_{53}=l_{55}^{+} \otimes l_{53}^{-} \\
& t_{54}=l_{55}^{+} \otimes l_{54}^{-} \\
& t_{55}=l_{55}^{+} \otimes l_{55}^{-} \\
& t_{1}
\end{aligned}
$$

or, equivalently 


$$
\begin{aligned}
& t_{11}=l_{11}^{-} \otimes l_{11}^{+}, \\
& t_{12}=l_{11}^{-} \otimes l_{12}^{+} \\
& t_{13}=l_{11}^{-} \otimes l_{13}^{+} \\
& t_{14}=l_{11}^{-} \otimes l_{14}^{+} \\
& t_{15}=l_{11}^{-} \otimes l_{15}^{+} \\
& t_{21}=l_{21}^{-} \otimes l_{11}^{+} \\
& t_{22}=l_{21}^{-} \otimes l_{12}^{+}+l_{22}^{-} \otimes l_{22}^{+} \\
& t_{23}=l_{21}^{-} \otimes l_{13}^{+}+l_{22}^{-} \otimes l_{23}^{+} \\
& t_{24}=l_{21}^{-} \otimes l_{14}^{+}+l_{22}^{-} \otimes l_{24}^{+} \\
& t_{25}=l_{21}^{-} \otimes l_{15}^{+}+l_{22}^{-} \otimes l_{25}^{+} \\
& t_{31}=l_{31}^{-} \otimes l_{11}^{+} \\
& t_{32}=l_{31}^{-} \otimes l_{12}^{+}+l_{32}^{-} \otimes l_{22}^{+}, \\
& t_{33}=l_{31}^{-} \otimes l_{13}^{+}+l_{32}^{-} \otimes l_{23}^{+}+l_{33}^{-} \otimes l_{33}^{+}, \\
& t_{34}=l_{31}^{-} \otimes l_{14}^{+}+l_{32}^{-} \otimes l_{24}^{+}+l_{33}^{-} \otimes l_{34}^{+}, \\
& t_{35}=l_{31}^{-} \otimes l_{15}^{+}+l_{32}^{-} \otimes l_{25}^{+}+l_{33}^{-} \otimes l_{35}^{+}, \\
& t_{41}=l_{41}^{-} \otimes l_{11}^{+}, \\
& t_{42}=l_{41}^{-} \otimes l_{12}^{+}+l_{42}^{-} \otimes l_{22}^{+}, \\
& t_{43}=l_{41}^{-} \otimes l_{13}^{+}+l_{42}^{-} \otimes l_{23}^{+}+l_{43}^{-} \otimes l_{33}^{+}, \\
& t_{44}=l_{41}^{-} \otimes l_{14}^{+}+l_{42}^{-} \otimes l_{24}^{+}+l_{43}^{-} \otimes l_{34}^{+}+l_{44}^{-} \otimes l_{44}^{+}, \\
& t_{45}=l_{41}^{-} \otimes l_{15}^{+}+l_{42}^{-} \otimes l_{25}^{+}+l_{43}^{-} \otimes l_{35}^{+}+l_{44}^{-} \otimes l_{45}^{+}, \\
& t_{51}=l_{51}^{-} \otimes l_{11}^{+}, \\
& t_{52}=l_{51}^{-} \otimes l_{12}^{+}+l_{52}^{-} \otimes l_{22}^{+}, \\
& t_{53}=l_{51}^{-} \otimes l_{13}^{+}+l_{52}^{-} \otimes l_{23}^{+}+l_{53}^{-} \otimes l_{33}^{+}, \\
& t_{54}=l_{51}^{-} \otimes l_{14}^{+}+l_{52}^{-} \otimes l_{24}^{+}+l_{53}^{-} \otimes l_{34}^{+}+l_{54}^{-} \otimes l_{44}^{+}, \\
& t_{55}=l_{51}^{-} \otimes l_{15}^{+}+l_{52}^{-} \otimes l_{25}^{+}+l_{53}^{-} \otimes l_{35}^{+}+l_{54}^{-} \otimes l_{45}^{+}+l_{55}^{-} \otimes l_{55}^{+} \\
& t_{1}
\end{aligned}
$$

It is straightforward to verify that the matrix $T$ defined above really satisfies all relations which a quantum group should be satisfied. Let $P^{\otimes}$ be the transposition operator, for arbitrary matrices $A$ and $B$, which satisfies

$$
P^{\otimes}: \quad A \dot{\otimes} B=B \dot{\otimes} A .
$$

Then the comultiplication $\Delta$ for these tensor operator $T$ can be introduced as

$$
\Delta=\left(\mathrm{id} \otimes P^{\otimes} \otimes \mathrm{id}\right) \Delta_{L^{ \pm}} \dot{\otimes} \Delta_{L^{\mp}} .
$$

It is easy to check that

$$
\begin{aligned}
\Delta(T) & =\left(\mathrm{id} \otimes P^{\otimes} \otimes \mathrm{id}\right) \Delta_{L^{ \pm}} \dot{\otimes} \Delta_{L \mp}\left(L^{ \pm} \dot{\otimes} L^{\mp}\right) \\
& =\left(\mathrm{id} \otimes P^{\otimes} \otimes \mathrm{id}\right)\left(L^{ \pm} \dot{\otimes} L^{ \pm} \dot{\otimes} L^{\mp} \dot{\otimes} L^{\mp}\right) \\
& =\left(L^{ \pm} \dot{\otimes} L^{\mp}\right) \dot{\otimes}\left(L^{ \pm} \dot{\otimes} L^{\mp}\right) \\
& =T \dot{\otimes} T .
\end{aligned}
$$


Define counit operator $\epsilon$ as

$$
\epsilon=\epsilon_{L^{ \pm}} \otimes \epsilon_{L^{\mp}} .
$$

Then we have $\epsilon(T)=1$. Finally, the antipode operator $S$ is of the form,

$$
S=P\left(S_{L^{\mp}} \otimes S_{L^{ \pm}}\right) P^{\otimes} .
$$

By making use of the relations (35), it is a straightforward calculation to check that

$$
\begin{aligned}
S(T) & =S\left(L^{ \pm} \dot{\otimes} L^{\mp}\right) \\
& =P\left(S_{L^{\mp}} \otimes S_{L^{ \pm}}\right) P^{\otimes}\left(L^{ \pm} \dot{\otimes} L^{\mp}\right) \\
& =P\left(S_{L^{\mp}}\left(L^{\mp}\right) \otimes S_{L^{ \pm}}\left(L^{ \pm}\right)\right) \\
& =C T^{t} C^{-1} .
\end{aligned}
$$

At the stage, we can say that a realization of quantum group $S O_{q}(5)$ is given in terms of quantum algebra $U_{q}(s o(5))$.

\section{Quantum AdS Algebra}

Quantum field theory is usually constructed based on representations of an algebra. To get a quantum field theory on noncommutative geometry, we should investigate properties of the quantum AdS algebra. Of course, first of all, we should give conjugations on quantum AdS algebra, which is consistent with conjugations on quantum AdS group and quantum AdS space.

At the case of $|q|=1$, conjugation on quantum AdS group is given by

$$
\begin{aligned}
T^{*} & =\mathcal{D T D}^{-1} \\
& =\left(\begin{array}{ccccc}
t_{11} & t_{12} & -t_{13} & t_{14} & t_{15} \\
t_{21} & t_{22} & -t_{23} & t_{24} & t_{25} \\
-t_{31} & -t_{32} & t_{33} & -t_{34} & -t_{35} \\
t_{41} & t_{42} & -t_{43} & t_{44} & t_{45} \\
t_{51} & t_{52} & -t_{53} & t_{54} & t_{55}
\end{array}\right) .
\end{aligned}
$$

From the relationship between quantum group and quantum algebra, i.e., $T=L^{ \pm} \dot{\otimes} L^{\mp}$, we deduce directly the corresponding quantum AdS algebra as

$$
\begin{aligned}
& X_{1}^{+*}=X_{1}^{+}, \quad X_{1}^{-*}=X_{1}^{-}, \quad H_{1}^{*}=-H_{1}, \\
& X_{2}^{+*}=-X_{2}^{+}, \quad X_{2}^{-*}=-X_{2}^{-}, \quad H_{2}^{*}=-H_{2} .
\end{aligned}
$$


It is easy to show that this type of conjugation on quantum algebra satisfies all requirements for a conjugation.

For the case of $q$ being real, we know from the previous section that the conjugation structure of the quantum AdS group is given by

$$
\begin{aligned}
T^{*}= & \mathcal{N} C^{t} T\left(C^{-1}\right)^{t} \mathcal{N}^{-1} \\
= & \left(\begin{array}{ccccc}
t_{55} & -q t_{54} & -q^{3 / 2} t_{53} & -q^{2} t_{52} & q^{3} t_{51} \\
-q^{-1} t_{45} & t_{44} & q^{1 / 2} t_{43} & q t_{42} & -q^{2} t_{41} \\
-q^{-3 / 2} t_{35} & q^{-1 / 2} t_{34} & t_{33} & q^{1 / 2} t_{32} & -q^{3 / 2} t_{31} \\
-q^{-2} t_{25} & q^{-1} t_{24} & q^{-1 / 2} t_{23} & t_{22} & -q t_{21} \\
q^{-3} t_{15} & -q^{-2} t_{14} & -q^{-3 / 2} t_{13} & -q^{-1} t_{12} & t_{11}
\end{array}\right) .
\end{aligned}
$$

For $T=L^{ \pm} \dot{\otimes} L^{\mp}$, the proper conjugation operation is given by

$$
T^{*}=L^{\mp *} \dot{\otimes} L^{ \pm *}, \quad\left(L^{ \pm *}\right)_{i j} \equiv\left(L_{i j}^{ \pm}\right)^{*} .
$$

Comparing of Eq.(42) and (43) gives the corresponding quantum AdS algebra to quantum AdS group and quantum AdS space,

$$
\begin{array}{lll}
X_{1}^{+*}=-q^{2} X_{1}^{-}, & X_{1}^{-*}=-q^{-2} X_{1}^{+}, & H_{1}^{*}=H_{1}, \\
X_{2}^{+*}=q^{3 / 2} X_{2}^{-}, & X_{2}^{-*}=q^{-3 / 2} X_{2}^{+}, & H_{2}^{*}=H_{2} .
\end{array}
$$

Therefore, finally, we obtain the quantum AdS algebra for both the case of $|q|=1$ and

$q \in R$. The representations of the quantum AdS algebra 18 should be foundations of constructing quantum field theory on the quantum AdS space. Further investigating on this direction is still on progressing.

\section{Acknowledgments}

I would like to thank Prof. J. Wess for introducing the problem to me and for enlightening discussions. I am grateful to B. L. Cerchiai and H. Steinacker for valuable discussions. The work was supported in part by the National Science Foundation of China under Grant 19625512.

\section{References}

[1] C. Fronsdal, Rev. Mod. Phys. 37, 221 (1965); Phys. Rev. D10, 589 (1974). 
[2] D. Z. Freedmen and A. Das, Phys. Lett. B74, 333 (1977); S. M. Christensen, M. J. Duff, G. W. Gibbons and M. Rocek, Phys. Rev. Lett. 45, 161 (1980).

[3] J. Maldacena, Adv. Theor. Math. Phys. 2, 231 (1998).

[4] S. Gubser, I. Klebanov and A. Polyakov, Phys. Lett. B428, 105 (1998).

[5] E. Witten, Adv. Theor. Math. Phys. 2, 253 (1998).

[6] M. Green, J. H. Schwarz and E. Witten, "Superstring Theory", (2 volumes), Cambridge University Press (1986).

[7] J. H. Schwarz, Lectures on Superstring and M Theory Dualities, Nucl. Phys. (Proc. Suppl.) 55B, 1 (1997).

[8] T. Banks, W. Fischler, S. H. Shenker, and L. Susskind, Phys. Rev. D55, 5112 (1997).

[9] L. D. Faddeev, N. Yu. Reshetikhin and L. A. Takhtajan, Leningrad Math. J. 1, $193(1990)$.

[10] J. Wess and B. Zumino, Nucl. Phys. (Proc. Suppl.) 18B, 302 (1990).

[11] U. Carow-Watamura, M. Schlieker, and S. Watamura, Z. Phys. C 49, 439 (1991).

[12] O. Ogievetsky, Lett. Math. Phys. 24, 245 (1992).

[13] G. Fiore, Commun. Math. Phys. 169, 475 (1995).

[14] Z. Chang, Physics Reports 262, 137 (1995).

[15] P. Aschieri, Real forms of quantum orthogonal quantum groups, q-Lorents groups in any dimension, preprint LBNL-41692 (1998), math.QA/9805120.

[16] O. Ogievetsky and B. Zumino, Lett. Math. Phys. 25, 121 (1992).

[17] Z. Chang, H. Y. Guo and K. Wu, J. Phys. A31, L495 (1998).

[18] H. Steinacker, Commun. Math. Phys. 192, 687 (1998). 